\title{
Avalanche dynamics of fiber bundle models
}

\author{
R. C. Hidalgo, ${ }^{1}$ F. Kun, ${ }^{2}$ K. Kovács, ${ }^{2}$ and I. Pagonabarraga ${ }^{3}$ \\ ${ }^{1} A M A D E$, Departament de Física, Universitat de Girona, 17071 Girona, Spain \\ ${ }^{2}$ Department of Theoretical Physics, University of Debrecen, P.O. Box 5, H-4010 Debrecen, Hungary \\ ${ }^{3}$ Departament de Física Fonamental, Universitat de Barcelona, 08028 Barcelona, Spain
}

(Received 11 May 2009; published 10 November 2009)

\begin{abstract}
We present a detailed analytical and numerical study of the avalanche distributions of the continuous damage fiber bundle model (CDFBM). Linearly elastic fibers undergo a series of partial failure events which give rise to a gradual degradation of their stiffness. We show that the model reproduces a wide range of mechanical behaviors. We find that macroscopic hardening and plastic responses are characterized by avalanche distributions, which exhibit an algebraic decay with exponents between $5 / 2$ and 2 different from those observed in mean-field fiber bundle models. We also derive analytically the phase diagram of a family of CDFBM which covers a large variety of potential avalanche size distributions. Our results provide a unified view of the statistics of breaking avalanches in fiber bundle models.
\end{abstract}

DOI: 10.1103/PhysRevE.80.051108

PACS number(s): 05.20.Dd, 46.50.+a

\section{INTRODUCTION}

The damage and fracture of heterogeneous materials are very interesting scientific problems with a broad spectrum of technological applications [1-3]. It is well known that under a constant or slowly increasing external load, the fracture of heterogeneous materials proceeds in bursts, i.e., local breakings occur in correlated avalanches separated by silent periods [4-15]. Since the bursting activity generates elastic waves, it can be recorded by means of the acoustic emission technique, the primary source of information on the microscopic dynamics of fracture. Recently, experiments on a large variety of materials with disordered microstructure have pointed out that the amplitude and energy distributions of acoustic signals, as well as the distribution of delay time intervals between consecutive emissions are characterized by power laws, where the exponents show a certain degree of universality [4-13].

Among the theoretical approaches to the problem, the fiber bundle model (FBM) plays a crucial role, since it captures the main ingredients of the fracture of disordered materials but it is still simple enough to facilitate analytical calculations [16-31]. In FBM the specimen is discretized in terms of parallel fibers which are subject to a longitudinal external load. The fibers have identical elastic properties but stochastically distributed breaking thresholds. While quasistatically increasing the external load, the weaker fiber breaks in the bundle. The load of the broken fiber must be overtaken by the intact fibers whose local load may also exceed their respective failure threshold and also fail. Hence, a single breaking event may induce an entire avalanche, which goes on until the avalanche stops or destroys the entire system. It has been shown in the framework of FBM that in the limit of equal load sharing, the size distribution of bursts is characterized by a universal power-law decay with exponent $5 / 2$ for a broad class of disorder distributions [17-22]. It was shown analytically that when bursts are recorded solely in the vicinity of the critical point of macroscopic failure, a crossover occurs to a lower value of the exponent $3 / 2$. The crossover of the bursts size exponent when approaching the critical point addresses the possibility to design techniques to forecast the imminent failure event $[22,25,26]$.

For theoretical studies on fracture statistics, it is a great challenge to understand the origin of the scale free bursting activity, to reveal the role of the underlying disorder and of the breaking mechanisms, and to explore the possible universality classes which characterize the fracture process $[21,22,28-31]$. Recently, we have shown by analytical and numerical means that mixing fibers of strongly different strength, i.e., when the bundle is composed of two subsets of fibers, where one subset is unbreakable, the avalanche size distribution exhibits a transition from the well-known exponent $5 / 2$ to a lower one $9 / 4$ when the mixing ratio surpasses a threshold value [23].

In the present paper we investigate the effect of the breaking mechanism of fibers on avalanche statistics based on a continuous damage fiber bundle model (CDFBM), introduced recently [32-34]. In CDFBM the fibers do not suffer instantaneous failure when the local load exceeds their breaking threshold; instead, they loose their stiffness gradually in a sequence of partial breaking events. The gradual breaking sequence is characterized by two parameters: the fraction of stiffness kept by the fiber after a partial breaking, and the total number of allowed breakings. Varying the two parameters, the model provides various types of mechanical responses from simple quasibrittle behavior through plasticity to hardening. We demonstrate by analytical calculations that depending on the details of the constitutive curve, the size distribution of bursts has a power-law functional form with a spectrum of exponents between $\frac{5}{2}$ and 2 . We construct analytically the phase diagram of the model, which provides an overview of all possible avalanche behaviors, and verify the analytical predictions with computer simulations.

\section{CONTINUOUS DAMAGE FIBER BUNDLE MODEL}

The continuous damage fiber bundle model has been introduced recently [32-34] as an extension of the classical fiber bundle model $[16,18]$. The model consists of a set of $N$ linearly elastic fibers with identical Young modulus $E_{f}$ orga- 
nized into a parallel bundle. Under an increasing external load the fibers exhibit brittle failure, i.e., they have a linearly elastic behavior up to a critical load $\sigma_{t h}$ where they break. The fibers have an identical Young modulus $E_{f}$; however, the breaking threshold $\sigma_{t h}$ is a stochastic variable characterized by a probability density $p\left(\sigma_{t h}\right)$ and a distribution function $P\left(\sigma_{t h}\right)$. The fiber bundle is loaded uniaxially, giving rise to a global deformation $f$, which is related to the applied load through $\sigma=E_{f} f$. In the classical FBM, when fiber $i$ experiences a local load $\sigma_{i}$ larger than its strength threshold, $\sigma_{t h}^{i}$, it fails so that its stiffness is set to zero.

As a novel element, in CDFBM it is assumed that the failure of fibers is not instantaneous, instead the fibers undergo a gradual degradation process. When the local load of fiber $i$ reaches its breaking threshold $\sigma_{t h}^{i}$ it suffers only partial failure such that its stiffness $E_{f}$ is reduced by a factor 0 $\leq \alpha \leq 1$. When the external load is further increased the fiber again exhibits a linear elastic behavior but with a lower value of the Young modulus $\alpha E_{f}$, and hence, it can fail again in the same manner as before. The maximum number of breaking events $k_{\max }$ that the fibers can suffer together with the stiffness reduction factor $\alpha$ is very important parameters of CDFBM. Thus, given that $\alpha^{k} E_{f}$ is the remaining stiffness of a single fiber, which has experienced $k$ failure events, $a^{k_{\max }} E_{f}$ can be interpreted as the maximum level of degradation of the fibers' elastic properties. The limiting case $k_{\max }=\infty$ represents the plastic limit of the model.

Moreover, it is important to note that once a fiber has failed it can either keep the same strength threshold, which corresponds to a quenched distribution of failure strengths, or choose a different strength value from the prescribed threshold distribution, corresponding to an annealed distribution of failure strengths. It has been presented in Refs. [32-34] that depending on the way how the stiffness of fibers is treated after $k_{\max }$ number of failure events, the model can describe both macroscopic hardening and macroscopic fracture of the bundle. Hardening behavior is obtained when the fibers retain their $\alpha^{k_{\max }} E_{f}$ stiffness after having failed $k_{\max }$ times. In this case the macroscopic constitutive equation of the system converges to an asymptotic linear behavior. Setting the stiffness of fibers to zero after $k_{\max }$ breakings implies the complete failure of the fibers, which then leads to macroscopic fracture of the entire bundle instead of hardening. In the present paper our study is restricted to the case of quenched disorder and macroscopic hardening in the final state.

When the fibers are allowed to break only once $k_{\max }=1$, the constitutive equation of the fiber bundle subject to an external force $F$ can simply be expressed as

$$
\sigma=\frac{F}{N}=f[1-P(f)]+\alpha f P(f),
$$

where $P(f)$ and $1-P(f)$ are the fraction of failed and intact fibers, respectively, and the Young modulus $E_{f}$ of intact fibers is taken to be unity $E_{f}=1$. In Eq. (1) the first term provides the load carried by intact fibers while the second term is the load-bearing contribution of the failed ones. It can be seen that for $\alpha=0$, the constitutive behavior of CDFBM
Eq. (1) reduces to the classical fiber bundle model where failed fibers do not carry any load [16-18,22,30].

It is worth noting that Eq. (1) can be rewritten in the form

$$
\sigma=(1-\alpha) f[1-P(f)]+\alpha f,
$$

which allows for an interesting alternative physical interpretation: when fibers are allowed to break only once but they retain their reduced Young modulus, the constitutive behavior of CDFBM is identical to another fiber bundle, which is composed of two subsets of fibers with widely different strength characteristics but identical elastic behavior. A subset of $\alpha N$ fibers is unbreakable while the remaining (1 $-\alpha) N$ fibers are breakable characterized by a threshold distribution $P$ [23]. Very recently, we have demonstrated that the presence of unbreakable fibers results into a complex behavior of the system; especially the avalanche statistics presents substantial changes compared to the simple FBM [23]. These former results imply that in CDFBM an even richer behavior of the avalanche statistics can be expected, which may provide a realistic description of certain materials.

For the general case where fibers are allowed to fail $k_{\max }$ times and the fibers keep their final stiffness $\alpha^{k_{\max }} E_{f}$ after having failed $k_{\max }$ times, the constitutive equation can be cast into the form

$$
\begin{aligned}
\sigma= & f[1-P(f)]+\sum_{i=1}^{k_{\max }-1} \alpha^{i} f\left[P\left(\alpha^{i-1} f\right)-P\left(\alpha^{i} f\right)\right] \\
& +\alpha^{k_{\max }} f P\left(\alpha^{k_{\max }-1} f\right) .
\end{aligned}
$$

In Eq. (3) $f(1-P(f)$ is the load carried by the intact fibers, while $\alpha^{i} f\left[P\left(\alpha^{i-1} f\right)-P\left(\alpha^{i} f\right)\right]$ and $\alpha^{k_{\max }} f P\left(\alpha^{k_{\max }-1} f\right)$ account for the load on the fibers that have failed $i$ times and $k_{\max }$ times, respectively.

To be specific, throughout the paper we will consider the Weibull distribution

$$
P(\sigma)=1-\exp \left[-\left(\sigma / \sigma_{o}\right)^{m}\right]
$$

to characterize the fiber breaking thresholds, where $\sigma_{o}$ denotes the characteristic fiber strength and $m$ is the Weibull exponent. The constitutive behavior of Eq. (3) for this Weibull distribution is illustrated in Figs. 1 and 2. The figures show that the remaining fiber load-bearing capacity confers the material with elastic hardening. In fact, once all fibers have failed, the constitutive curve $\sigma(f)$ converges to an asymptotic straight line and the system is characterized by an elastic constant $\alpha^{k_{\max } E_{f}}$. As $k_{\max }$ increases, the asymptotic linear behavior of hardening is getting weaker and it is preceded by an increasingly wider plateau. The larger $k_{\max }$ is, the flatter the constitutive curve becomes; in the limiting case of $k_{\max } \rightarrow \infty$ the macroscopic material response becomes completely plastic. At a given value of $k_{\max }$ the shape of $\sigma(f)$ along the plateau regime can be controlled by the stiffness reduction parameter $\alpha$. For low values of $\alpha$, local maxima and minima can be observed. For a given $k_{\max }$ there exists always a critical value $\alpha_{c}$ of the stiffness reduction factor for which the wavy shape of Fig. 1 disappears, and in the parameter regime $\alpha>\alpha_{c}\left(k_{\max }\right)$ the plateau becomes monotonous and essentially flat. The critical value $\alpha_{c}\left(k_{\max }\right)$ and the 


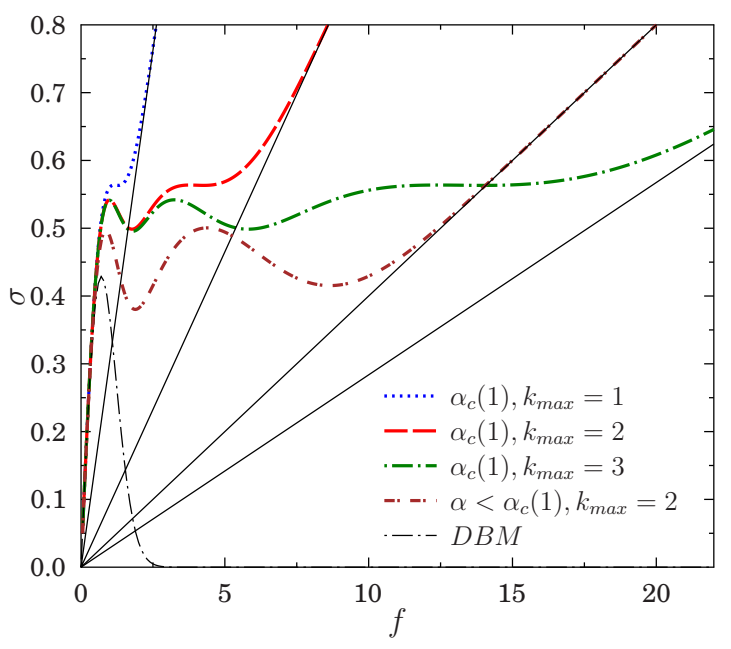

FIG. 1. (Color online) Constitutive behavior of the continuous damage fiber bundle model (CDFBM) for a fixed value of the stiffness reduction parameter $\alpha=\alpha_{c}\left(k_{\max }=1\right)$ on increasing the maximum number of allowed failures, $k_{\max }$.

shape of the constitutive curve $\sigma(f)$ in the vicinity of this critical point play an essential role in the statistics of bursts. Figure 2 presents the constitutive curves $\sigma(f)$ for different $k_{\max }$ at the corresponding critical value of $\alpha$. It can be observed that the larger $k_{\max }$ is, the wider the plastic plateau turns.

The constitutive relation Eq. (3) can also be expressed as a relation between the applied load $\sigma$ and the macroscopic deformation of the bundle $f$ experienced by each fiber,

$$
\sigma=Y(f) f
$$

where $Y(f)$ defines the effective Young modulus of the system at deformation $f$,

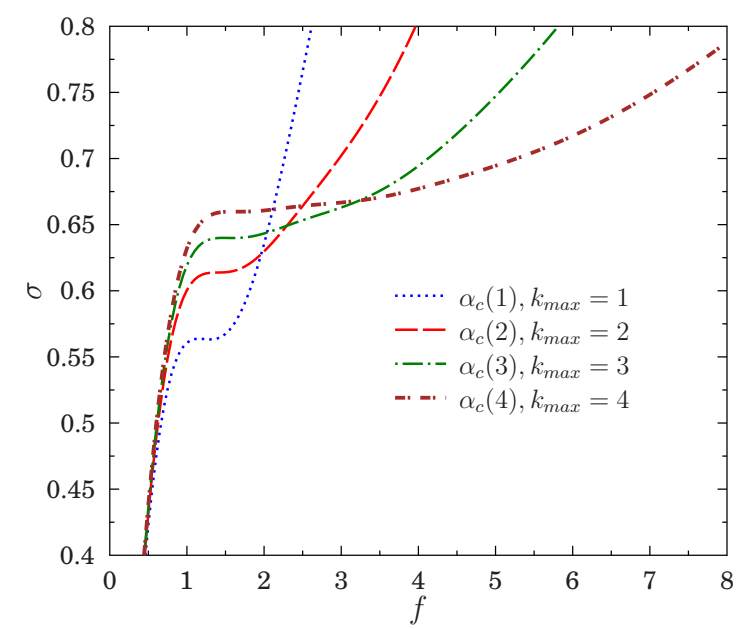

FIG. 2. (Color online) Constitutive curves of CDFBM varying the maximum number of allowed failures $k_{\max }$ at the corresponding critical value of the stiffness reduction factor $\alpha$. As $k_{\max }$ increases the plastic plateau gets wider. The failure thresholds are Weibull distributed with the parameter values $m=2$ and $\sigma_{o}=1$.

$$
\begin{aligned}
Y(f)= & {[1-P(f)]+\sum_{i=1}^{k_{\max }-1} \alpha^{i}\left[P\left(\alpha^{i-1} f\right)-P\left(\alpha^{i} f\right)\right] } \\
& +\alpha^{k_{\max }} P\left(\alpha^{k_{\max }-1} f\right)
\end{aligned}
$$

in units of $E_{f}$.

Quasistatic loading of the bundle can be carried out in such a way that the load is incremented until a single fiber breaks. Then the load of the broken fiber is overtaken by the remaining intact ones which might induce further breaking. The subsequent breaking and load redistribution steps can result in an entire avalanche of breakings. In order to understand the statistics of burst of breaking fibers, let us consider the case when a fiber, which has broken $j$ times until the deformation $f$ was achieved, fails again. We have shown in Ref. [34] that the probability density function $p_{j}^{j+1}(f)$ of this event can be obtained in the form

$$
p_{j}^{j+1}(f)=\alpha^{j} p\left(\alpha^{j} f\right)
$$

As a consequence, the Young modulus of the breaking fiber gets reduced which releases stress distributed among the fibers. This stress redistribution leads to an increase in the global deformation $f$ which can be expressed as

$$
\delta f_{j}=\frac{\alpha^{j}(1-\alpha) f}{Y(f)},
$$

where index $j$ expresses that this strain increment appears as a consequence of a failure of fiber which has been broken $j$ times before. Using Eqs. (7) and (8), the total probability that a fiber breaks as a consequence of a fiber breaking when the material is subject to a strain $f$ reads as

$$
p_{\text {tot }}=\sum_{j=0}^{k-1} \delta f_{j} p_{j}^{j+1}(f)=\frac{(1-\alpha) f^{k-1}}{Y(f)} \sum_{j=0}^{2 j} \alpha^{2 j} p\left(\alpha^{j} f\right) .
$$

Using the expression for the Young modulus, and the fact that its derivative

$$
\frac{d Y(f)}{d f}=(1-\alpha) \sum_{j=1}^{k-1} \alpha^{2 j} p\left(\alpha^{j} f\right)
$$

is closely related to the overall breaking probability $p_{t o t}(f)$, we can express $p_{t o t}$ in the simple form

$$
p_{t o t}(f)=-\frac{f Y^{\prime}(f)}{Y(f)}
$$

Since the extrema of the constitutive curve satisfy $f Y^{\prime}(f)$ $+Y(f)=0$, in these points necessarily $p_{t o t}=1$ holds, which leads to the breaking of a macroscopic fraction of the sample, consistent with the $k_{\max }=1$ case studied in Ref. [23].

\section{AVALANCHE DYNAMICS}

Under a quasistatically increasing external load, the damage and fracture of heterogeneous materials proceed in a large number of events consisting of crack nucleation and sudden advancement of growing cracks. This process is accompanied by the release of elastic waves which can be re- 
corded by the acoustic emission technique. Hence, acoustic signals provide very valuable information about microscopic fracture dynamics in heterogeneous materials. In fiber bundle models, single fiber breakings can trigger an avalanche of breaking events which corresponds to the acoustic bursts of the experiments. One of the main advantages of FBMs is that they allow for the analytic derivation of the statistics of breaking bursts at least in the limit of global load sharing.

In the global load sharing limit of simple FBM, Hansen and Hemmer $[17,18]$ proved that the probability density $D(\Delta)$ to observe an avalanche of size $\Delta$, during a quasistatic loading process, has the form

$$
D(\Delta)=\frac{\Delta^{\Delta-1}}{\Delta !} \int_{0}^{f_{m}} p(f)\left(1-a_{f}\right) a_{f}^{\Delta-1} e^{-a_{f} \Delta} d f \equiv \frac{\Delta^{\Delta-1}}{\Delta !} I(\Delta),
$$

where $a_{f}$ is the average fraction of fibers which breaks as a result of an infinitesimal increase in the applied deformation $f$. For CDFBM $a_{f}$ is identical to the total breaking probability $a_{f}=p_{\text {tot }}$ given by Eq. (11), which can further be simplified to the form

$$
a_{f}=-\frac{d \ln Y(f)}{d \ln f} .
$$

In the following we provide an analytic derivation of the burst size distribution of CDFBM starting from Eqs. (12) and (13).

For convenience, we introduce the auxiliary function $I(\Delta)$ with the definition

$$
I(\Delta) \equiv \int_{0}^{f_{m}} p(f) \frac{1-a_{f}}{a_{f}} e^{-\Delta\left[a_{f}-\ln a_{f}\right]} d f .
$$

We are interested in large burst events, and hence will concentrate on the region of large avalanches $(\Delta \gg 1)$. In this regime the Stirling equation $\ln n ! \simeq\left(n+\frac{1}{2}\right) \ln n-n+\ln \sqrt{2 \pi}$ is used and the avalanche distribution $D(\Delta)$ can be expressed as

$$
D(\Delta) \simeq \frac{e^{\Delta}}{\sqrt{2 \pi} \Delta^{3 / 2}} I(\Delta) .
$$

Under global load sharing conditions, which is equivalent to the CDFBM with $k_{\max }=1$ and $\alpha=0$, the upper integral limit in Eq. (14) is the location of the parabolic maximum of the constitutive curve $f_{m}$. Thus, for large $\Delta$ this integral is controlled by the maximum of the exponent in the integrand. The extreme condition of $\psi \equiv a_{f}-\ln a_{f}$ results in $\psi^{\prime}=a_{f}^{\prime}(1$ $\left.-\frac{1}{a_{f}}\right)=0$, according to a maximum at $a_{f}=1$. Carrying out the Taylor expansions of $a_{f}$ and $\psi_{f}$, it was shown in Refs. $[17,18]$ that the distribution $D(\Delta)$ simplifies to a power law

$$
D(\Delta) \sim \Delta^{-\tau},
$$

with the exponent $\tau=5 / 2$ for a broad class of disorder distributions, including the CDFBM with $k_{\max }=1$ and $\alpha=0$, where the constitutive curve of the system has a single quadratic maximum $[17,18]$.

Even when the asymptotic behavior of the avalanche distribution is controlled by the extremum of $\psi$, there may exist situations where the macroscopic constitutive curve of the system has an inflexion point. This is the case if $\psi^{\prime \prime}=0$ at the maximum, a situation which happens whenever $a_{f}^{\prime}=0$ for $a_{f}=1$. One then needs to continue the Taylor expansion of $\psi$ to identify the relevant leading contribution. Following the usual procedure, one arrives at

$$
I(\Delta) \simeq \frac{p\left(f_{c}\right) a_{f_{c}^{\prime \prime}}^{\prime} e^{-\Delta}}{2} \int_{0}^{\infty} d f\left(f-f_{c}\right)^{2} e^{\left[3 a_{f_{c}}^{\prime \prime 2} \Delta / 4 !\right]\left(f-f_{c}\right)^{4}} .
$$

which implies that the asymptotic decay of the avalanche size distribution is characterized by a different algebraic tail

$$
D(\Delta) \simeq \frac{\Gamma\left(\frac{3}{4}\right)}{24 \sqrt{3 \pi a_{f}^{\prime \prime}} 3^{1 / 4}} \Delta^{-9 / 4} .
$$

Figure 1 illustrates that such a scenario is found in the CDFBM with $k_{\max }=1$ when the stiffness reduction parameter $\alpha$ reaches its critical value $\alpha_{c}(1)$. As it has been discussed in Sec. II, for the maximum number of allowed breakings $k_{\max }=1$, at $\alpha=\alpha_{c}(1)$ the local quadratic maximum of the constitutive curve disappears, $\sigma(f)$ becomes monotonous and an inflection point is formed. The above analytic result Eq. (18) demonstrates that the presence of the inflexion point substantially changes the avalanche statistics of the system.

One can then generalize the analysis and consider a situation where the constitutive relation $\sigma(f)$ leads to an incremental average fiber breaking, $a_{f}$ which gives rise to a generalized inflexion point of order $n$ at the extremum of $\psi$ of the location $f_{m}$. Carrying out again a Taylor expansion of $\psi$ to leading order around its extremum, we arrive at

$$
I(\Delta) \simeq \frac{p\left(f_{c}\right) a_{f}^{\prime \prime} e^{-\Delta}}{2} \int_{0}^{\infty} d f\left(f-f_{m}\right)^{n} e^{\left[(2 n-1) a_{f}^{(n)^{n}} \Delta / 2 n !\right]\left(f-f_{m}\right)^{2 n}},
$$

where $a_{f_{m}}^{(n)}$ denotes the first nonvanishing derivative of $a_{f}$ at $f_{m}$. It follows that the cumulative avalanche size distribution decays algebraically as

$$
D(\Delta) \sim \Delta^{-(4 n+1 / 2 n)} .
$$

This analytic result demonstrates that as the order of the inflexion point $n$ increases, the size distribution of avalanches remains a power low but the exponent gradually decreases. In the limit of an infinitely large plastic plateau $n \rightarrow \infty$ the avalanche distribution will converge to $D(\Delta) \sim \Delta^{-2}$.

It is also interesting to analyze the statistics of avalanches around a prescribed neighborhood of a generalized local extremal point $\left(f_{m} ; \sigma_{m}\right)$ of the constitutive curve of order $n$ where $\frac{d^{i} \sigma}{d f^{i}}=0(i=1 \ldots n-1$ and $n \in Z)$. Recently, it has been shown that restricting the analysis to the vicinity of the parabolic maximum of the constitutive curve, the avalanche size distribution shows a crossover between two power-law regimes: the asymptotics of the distribution has an exponent $5 / 2$ while the distribution of small avalanches decays slower with the exponent $3 / 2$. Considering avalanches only at the maximum of $\sigma(f)$, a single power law remains with exponent $3 / 2[19-22]$. 
In the general case of a local extremal point $\left(f_{m} ; \sigma_{m}\right)$ of order $n$, the size distribution of avalanches originating in the strain interval $f_{0} \leq f \leq f_{m}$ can be cast into the form

$$
D\left(\Delta, f_{0}\right)=\frac{\Delta^{\Delta-1}}{\Delta !} \int_{f_{0}}^{f_{m}} p(f)\left(1-a_{f}\right) a_{f}^{\Delta-1} e^{-a_{f} \Delta} d f .
$$

Hence, the asymptotics of the distribution reads as

$$
D\left(\Delta, f_{0}\right) \sim \Delta^{-4 n+1 / 2 n}\left[\Gamma\left(\frac{3 n+1}{2 n}\right)-\Gamma\left(\frac{3 n+1}{2 n}, \frac{\Delta}{\Delta_{c}}\right)\right],
$$

where $\Gamma(s)$ and $\Gamma(s, x)$ are the complete and incomplete gamma functions, respectively [35]. In Eq. (22) the crossover avalanche size $\Delta_{c}$ scales with the strain interval as

$$
\Delta_{c} \sim \frac{1}{\left(f_{m}-f_{0}\right)^{2 n}} .
$$

Depending on the avalanche size we can then identify two regimes in the avalanche size distribution

$$
D\left(\Delta, f_{0}\right) \sim \begin{cases}\Delta^{-(3 / 2)}, & \text { if } \Delta \ll \Delta_{c} \\ \Delta^{-[(4 n+1) / 2 n]}, & \text { if } \Delta \gg \Delta_{c} .\end{cases}
$$

When the avalanches are recorded in the vicinity of a generalized local extreme where $\frac{d^{i} \sigma}{d f^{i}}=0 \quad(i=1 \ldots n-1$ and $n \in Z)$, we find that the power law has an exponent which also differs from the one characterizing the size distribution of all avalanches. This result generalizes previous findings reported in [19-22], where a constitutive behavior with a single parabolic maximum $n=1$ was examined.

\section{COMPUTER SIMULATIONS}

In order to obtain a deeper understanding of the microscopic breaking mechanism in CDFBM, we have developed a simulation technique and explored numerically the distribution of bursts of fiber failures in Ref. [34]. Under stresscontrolled loading conditions, the failure of each fiber is followed by a redistribution of load, which can induce further fiber breakings, resulting in an avalanche of failure events. Previously, we have analyzed numerically the avalanche statistics of the model as a function of the number of failures, $k_{\max }$, and of the stiffness reduction parameter, $\alpha$, setting up an approximate phase diagram based on computer simulations [34]. In the following, we will make use of the analytic results of Secs. II and III, to deduce analytically the complete phase diagram of CDFBM on the $\left(\alpha ; k_{\max }\right)$ plane classifying all possible functional forms of the burst size distributions. The analytic predictions are checked with computer simulations for a Weibull distribution of breaking thresholds Eq. (4) with parameters $m=2$ and $\sigma_{0}=1$. In the simulations the number of fibers is fixed $N=160000$ and the burst size distributions are averaged over two hundred samples.

Figure 1 shows that, after the initial elastic response, the constitutive curve of CDFBM displays a plateau regime which generically is nonmonotonic; different local extrema develop along the plateau. Varying the value of $\alpha$ and $k_{\max }$,

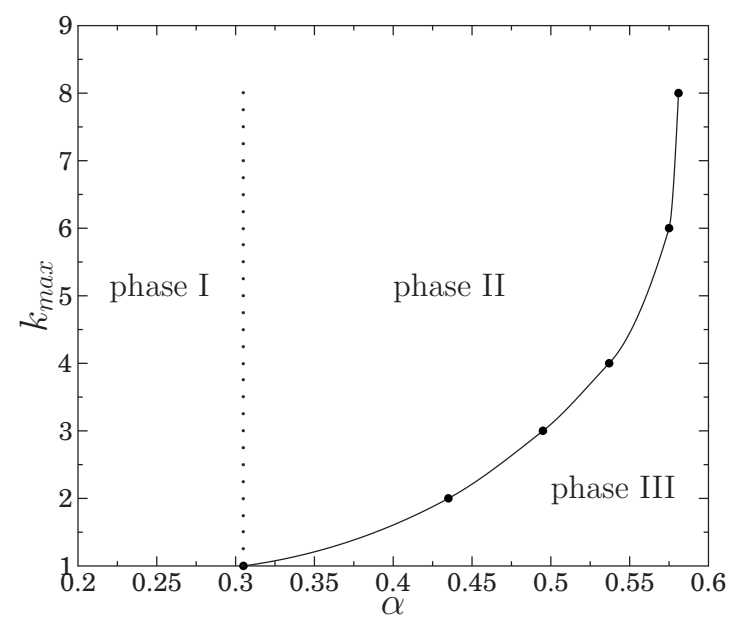

FIG. 3. Phase diagram of the continuous damage fiber bundle model, which determines the qualitatively different burst size distributions. The calculations of the phase boundaries have been carried out analytically for Weibull distributed breaking thresholds with $\sigma_{0}=1$ and $m=2$.

the number and type of the local extrema can be controlled. The above analytic calculations have shown that the qualitative shape of the local extrema has a substantial effect on the microscopic failure process. Especially, the asymptotics of the size distribution of breaking bursts is controlled by the mechanical response of the material near the local extrema and the last inflection point of the constitutive curves.

It is noticeable in Fig. 1 that the local extreme of the constitutive curves of the CDFBM are not parabolic in general, i.e., the larger the value of $k_{\max }$ is, the flatter the peaks are. When fibers can fail only once $k_{\max }=1$, below the critical stiffness reduction parameter, $\alpha<\alpha_{c}\left(k_{\max }=1\right)$, the constitutive curve, $\sigma(f)$, has one local maximum while above it $\sigma(f)$ becomes monotonous with an inflexion point. It can also be shown analytically that for $k_{\max }>1$ the constitutive curve has $k_{\max }$ local maxima; however, at the critical value $\alpha_{c}\left(k_{\max }=1\right)$ the last maximum turns into an inflexion point. This feature is illustrated in Fig. 1.

Carefully examining the model constitutive behavior, we have found that for $\alpha_{c}<\alpha_{c}(1)$ (independently of $k_{\max }$ ) the first parabolic maximum is always slightly higher than the rest of local maxima. Thus, the avalanche size distribution is determined just by the behavior of burst processes around the first parabolic maximum. Consequently, it results in an algebraic decay of the burst size distribution with an exponent $5 / 2$ similarly to simple FBMs. This scenario is represented by Phase I in the phase diagram of Fig. 3, where the burst distributions are described by Eq. (16) in agreement with Refs. [17,18]. The critical point $\alpha_{c}\left(k_{\max }=1\right)$ can be determined analytically as $\alpha_{c}(1)=m e^{-(1+m) / m} /\left[1+m e^{-(1+m) / m}\right]$ for a Weibull distribution. It reduces to $\alpha_{c}(1)=0.305$ for the parameter values $m=2$ and $\sigma_{0}=1$ and it is represented by the dotted vertical line in the phase diagram of Fig. 3 .

It has been shown in Sec. III that for each $k_{\max }$ there exists a critical value of the stiffness reduction parameter $\alpha_{c}\left(k_{\max }\right)$, above which the constitutive curve becomes monotonous, $d \sigma / d f \geq 0$ (see Fig. 2). In this case the avalanche statistics is determined by a generalized singular point $\left(f_{m} ; \sigma_{m}\right)$ of $\sigma(f)$ 
leading to a distinct behavior which is represented by Phase III in the phase diagram. The phase boundary in Fig. 3 separating Phase II and Phase III is the curve of $\alpha_{c}\left(k_{\max }\right)$. This curve was deduced by solving numerically $d \sigma / d f=0$ and $d^{2} \sigma / d f^{2}=0$ for a given $k_{\max }$ and looking for the minimum $\alpha_{c}\left(k_{\max }\right)$, which allowed for a common solution.

In this case the plateau region of the constitutive curve, $\sigma(f)$ does not have any maxima, and is characterized by the inflection region in $\sigma(f)$ around $\sigma_{m}$. In Sec. III we have shown analytically that in this situation the avalanche size distribution decays asymptotically as a power law with an exponent $D(\Delta) \sim \Delta^{-[(4 n+1) / 2 n]}$. The value of $n$ is directly related to the order of the first nonvanishing derivative of the constitute curve $\frac{d^{n} \sigma(f)}{d f^{n}} \neq 0$ at the generalized singular point $\left(f_{m} ; \sigma_{m}\right)$.

Note that the absence of local maxima in the constitutive curve $\sigma(f)$ in Phase III implies that the avalanche size distribution has an asymptotic exponential decay characterized by a correlation length, which diverges on approaching $\alpha_{c}$ from Phase III. In order to numerically verify the analytic predictions on the burst size distribution and to analyze the diverging correlation length when approaching the line $\alpha_{c}\left(k_{\max }\right)$, we have carried out computer simulations of CDFBM. The insets of Figs. 4(a) and 4(b) display the burst size distribution obtained numerically for $k_{\max }=2$ and $k_{\max }=4$ in the parameter range $\alpha>\alpha_{c}\left(k_{\max }\right)$, i.e., inside Phase III. The corresponding critical values can be obtained as $\alpha_{c}(2)=0.435$ and $\alpha_{c}(4)=0.537$. It can be seen in the insets of Figs. 4(a) and 4(b) that in agreement with the analytic predictions, the cutoff of the avalanche size distributions $D(\Delta)$ rapidly increases when $\alpha$ approaches $\alpha$ from above.

To further analyze the effect of varying $\alpha$ in Phase III, we introduce the scaling ansatz

$$
D(\Delta)=\bar{\Delta}_{\max }^{-\beta} g\left(\Delta / \bar{\Delta}_{\max }^{\xi}\right)
$$

for the avalanche size distribution, $D(\Delta)$, obtained above the critical point $\alpha>\alpha_{c}\left(k_{\max }\right)$. In Eq. (25), the mean value of the maximum avalanche, $\bar{\Delta}_{\max }$, is introduced as a scaling variable, while $\beta$ and $\xi$ are scaling exponents which must satisfy the relation $\beta=\tau \xi$. Figures 4(a) and 4(b) display the rescaled avalanche size distributions plotting $D(\Delta) \bar{\Delta}_{\max }^{\beta}$ as a function of $\Delta / \bar{\Delta}_{\max }^{\xi}$. The high-quality data collapse obtained with the parameters $\beta=3.12$ and $\xi=1.4$ are consistent with a powerlaw exponent $\tau=2.23$. Additionally, in Fig. 4(b) the collapsed data corresponding to $k_{\max }=4$ and $\alpha$ values just above $\alpha_{c}(4)$ is consistent with $\beta=3.28$ and $\xi=1.6$, which correspond to a power-law exponent $\tau=2.05$. It is noticeable that the size of plastic plateau of the constitutive curve increases with increasing $k_{\max }$. These results are consistent with the prediction that the exponent of the power law tends asymptotically to $\tau \rightarrow 2$ with increasing $k_{\max }$. This scenario corresponds to a situation where the plastic plateau of the constitutive relation becomes flatter as $k_{\max }$ increases. According to the theoretical description of the previous section, the exponent Eq. (20) characterizing the asymptotic decay of the cumulative avalanche size distribution can vary between $\frac{5}{2}$ when a local maximum controls the catastrophic avalanches, and 2 when
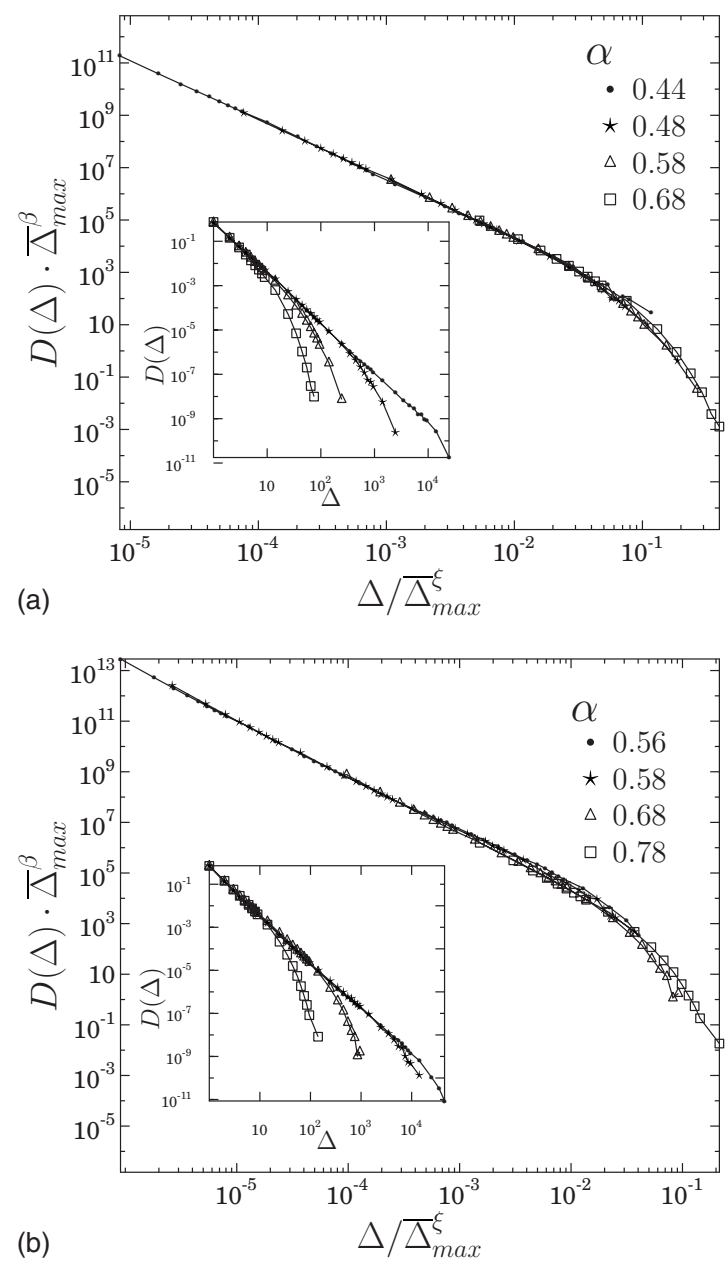

FIG. 4. Avalanche distributions obtained for a system with different maximum number of allowed failures: (a) $k_{\max }=2$ and (b) $k_{\max }=4$. Results for several values of $\alpha$ above $\alpha_{c}\left(k_{\max }\right)$ are shown. Good data collapse is obtained if the two axis are scaled according to the theoretical ansatz proposed in Eq. (25). The insets show the original avalanche size distributions.

the relevant region of the constitutive curve becomes sufficiently flat.

The fact that $\alpha_{c}(1)<\alpha<\alpha_{c}\left(k_{\max }\right)$ for $k_{\max }>1$ opens a region in the phase diagram where the constitutive curve is nonmonotonous (Phase II), where the first maximum of the constitutive curve is smaller than the subsequent ones. As a result, on increasing monotonously the applied stress the material explores the intermediate region of its constitutive curve where the material exhibits a nontrivial plastic behavior, where the characteristic avalanche activity is located in the neighborhood of the consecutive maxima. We can probe these subsequent bursting activities by analyzing the avalanche size distribution for different strain windows along the constitutive curve. In Fig. 5 we show the avalanche distributions until the first parabolic maximum, for two values of $k_{\max }$ displaying the generic algebraic decay $D(\Delta) \sim \Delta^{-5 / 2}$ with the usual mean-field exponent 5/2. Under stresscontrolled loading the valleys of the constitutive curve (as displayed in Fig. 1) cannot be accessed and the right-hand side of the different maxima does not contribute to the avalanche dynamics. However, we can analyze the avalanches 


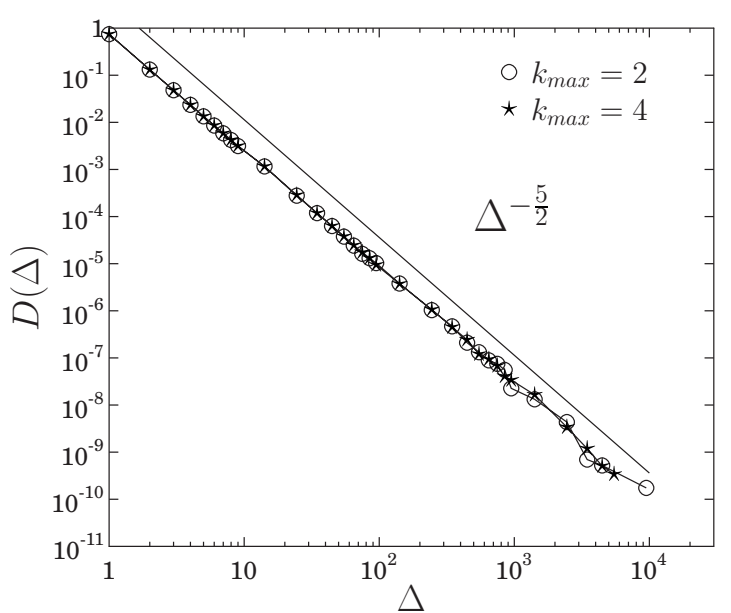

FIG. 5. Distribution of avalanches recorded from the beginning of the experiment till the first peak for $k_{\max }=2$ and $k_{\max }=4$. In both cases the value of $\alpha$ was chosen in the range $\alpha<\alpha_{c}(1)$. The continuous line corresponds to the analytical prediction $D(\Delta) \sim \Delta^{-5 / 2}$.

which propagate in the system in the neighborhood of the different maxima from bellow (slightly lower strain). As displayed in Fig. 6, in this case the avalanche has an asymptotic algebraic decay characterized by an exponent $-3 / 2$ independently of the maximum shape, as predicted from Eq. (24). We note that the crossover phenomenon of the avalanche size distribution $P(\Delta)$ predicted by Eqs. (23) and (24) can only be observed by setting different conditions on the strain value around the consecutive maxima of $\sigma(\varepsilon)$. Without precise conditioning, avalanches originating from different regimes of the consecutive curve mix up and the crossover behavior disappears. This is the reason why we do not analyze numerically the behavior of the crossover avalanche size $\Delta_{c}$ defined by Eq. (23).

Increasing $\alpha$ in Phase II, the maxima of the constitutive curve subsequently disappear, but in all cases the contribution to the largest avalanches is controlled by the last maxi-

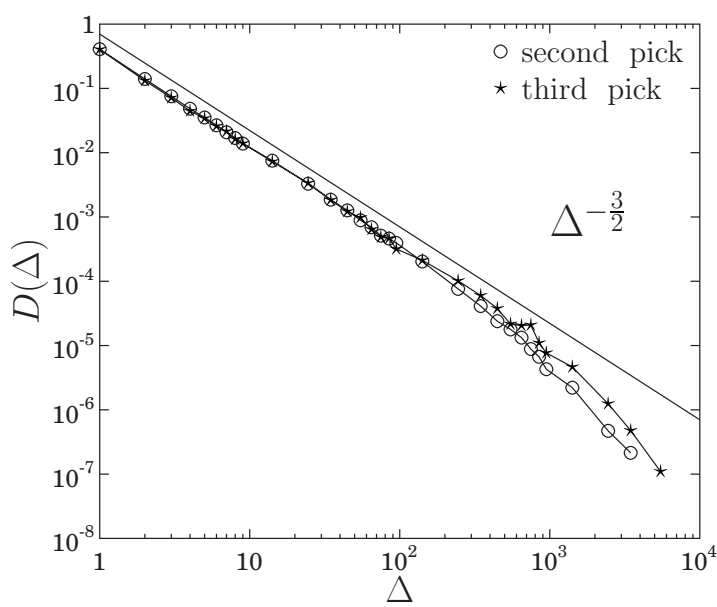

FIG. 6. Avalanche distributions in the vicinity of intermediate maxima of the constitutive curve from below, which develops for $\alpha>\alpha_{c}(1)$ for a maximum number of allowed failures $k_{\max }=4$ and $\alpha=0.40$. The continuous line corresponds to the theoretical prediction $D(\Delta) \sim \Delta^{-3 / 2}$. mum of the constitutive curve. As we approach $\alpha_{c}\left(k_{\max }\right)$, the last remaining maximum merges with the increasing part of the constitutive relation associated to material hardening, and right at the critical value the maximum gives rise to a plastic region in the constitutive curve characterized by a weak sensitivity to the applied strain. Mathematically, it can be regarded as a generalized inflection point, and accordingly, the asymptotic algebraic decay of the avalanche distribution function displays a peculiar exponent which cannot be smaller than 2 .

Our analysis holds for an unbounded distribution of breaking thresholds. For a uniform threshold distribution defined over a finite domain no inflexion points appear (independently of $\left.k_{\max }\right)$. Thus, in the phase diagram of Fig. 3 for the uniform distribution Phase I and Phase II merge into a single phase. Moreover, the behavior close to the transition line is simpler because the local extremum is always a single quadratic maximum; hence, the transition for any $k_{\max }$ has the same nature than in the case $k_{\max }=1$ [23]. We note that the avalanche statistics related to a single parabolic maximum notably differs when the system is loaded with finite load increments [36,37]. Nevertheless, the CDFBM for which the constitutive curve has more complex extreme points deserves a detailed study which will be presented elsewhere.

\section{DISCUSSION}

We have carried out a detailed study of the avalanche statistics of the continuous damage fiber bundle model where the fibers undergo a series of partial breaking events reducing gradually their stiffness. Slowly increasing the external load on the bundle, fibers break in bursts due to the subsequent load redistribution over intact fibers. The model has two main parameters, i.e., the stiffness reduction factor and the total number of allowed failures. Varying these parameters, the model captures various types of materials' response which can have also experimental relevance.

The plastic deformation of heterogeneous materials is known to proceed in avalanches on the microscale and recent experimental and theoretical investigations have revealed that dislocation rearrangements appear in bursts similarly to the microfracturing of heterogeneous materials $[14,15]$. The CDFBM shows that in a heterogeneous material macroscopic plasticity can emerge due to the subsequent partial failure without assuming plastic rheology of fibers. The microscopic dynamics and avalanche activity we obtain lie somewhere between microfracturing and plastic rearrangements. As the main outcome of the work, we have determined analytically the burst size distributions of the model and constructed a phase diagram of the system which characterizes all possible avalanche behaviors. We have shown that the presence of macroscopic hardening and plastic behavior result in burst distributions different from the usual mean-field result of FBM: power-law functional forms arise with an exponent varying between $5 / 2$ and 2 depending on the model parameters, which is then followed by an exponential cutoff. The 
analytic results have been verified by extensive computer simulations.

Although we have focused in a family of FBM where analytic progress has been possible, the results obtained are more general and indicate the intricate behavior and richness of highly disordered materials which display a nonmonotonous strain-load constitutive relation. The results obtained show that a careful understanding on the shape of the constitutive relation sheds light in the expected avalanche dynamics characterizing the failure process of materials on the microlevel.

\section{ACKNOWLEDGMENT}

This work is part of the Spanish-Hungarian Intergovernmental Scientific Project under Contract No. HH2005-0016. R.C.H. acknowledges the financial support of the Spanish Minister of Education and Science, through a Ramon y Cajal Program and the Project No. FIS2008-06034-C02-02. F.K. acknowledges the Janos Bolyai Fellowship of the Hungarian Academy of Sciences. I.P. thanks CAICYT (Grant No. FIS2008-04386) and DURSI (Distinción de la Generalitat de Catalunya) (Spain) for financial support.
[1] Statistical Models for the Fracture of Disordered Media, edited by H. J. Herrmann and S. Roux (Elsevier Science, North Holland, 1990).

[2] B. K. Chakrabarti and L. G. Benguigui, Statistical Physics of Fracture and Breakdown in Disordered Systems (Clarendon Press, Oxford, 1997).

[3] M. J. Alava, P. K. V. V. Nukala, and S. Zapperi, Adv. Phys. 55, 349 (2006).

[4] A. Petri, G. Paparo, A. Vespignani, A. Alippi, and M. Costantini, Phys. Rev. Lett. 73, 3423 (1994).

[5] A. Garcimartín, A. Guarino, L. Bellon, and S. Ciliberto, Phys. Rev. Lett. 79, 3202 (1997).

[6] A. Guarino, A. Garcimartín, and S. Ciliberto, Eur. Phys. J. B 6, 13 (1998).

[7] L. I. Salminen, A. I. Tolvanen, and M. J. Alava, Phys. Rev. Lett. 89, 185503 (2002).

[8] S. Deschanel, L. Vanel, G. Vigier, N. Godin, and S. Ciliberto, Int. J. Fract. 140, 87 (2006).

[9] S. Deschanel, L. Vanel, N. Godin, and S. Ciliberto, J. Stat. Mech. (2009) P01018.

[10] D. Bonamy, S. Santucci, and L. Ponson, Phys. Rev. Lett. 101, 045501 (2008).

[11] H. Nechad, A. Helmstetter, R. El Guerjouma, and D. Sornette, Phys. Rev. Lett. 94, 045501 (2005).

[12] M. B. J. Meinders and T. van Vliet, Phys. Rev. E 77, 036116 (2008).

[13] M. R'Mili, M. Moevus, and N. Godin, Compos. Sci. Technol. 68, 1800 (2008).

[14] M. C. Miguel, A. Vespignani, S. Zapperi, J. Weiss, and J. R. Grasso, Nature (London) 410, 667 (2001).

[15] M. C. Miguel and S. Zapperi, Science 312, 1151 (2006).

[16] H. E. Daniels, Proc. R. Soc. London, Ser. A 183, 405 (1945).

[17] A. Hansen and P. C. Hemmer, Phys. Lett. A 184, 394 (1994).

[18] M. Kloster, A. Hansen, and P. C. Hemmer, Phys. Rev. E 56, 2615 (1997).
[19] S. Pradhan and B. K. Chakrabarti, Phys. Rev. E 65, 016113 (2001).

[20] S. Pradhan, A. Hansen, and P. C. Hemmer, Phys. Rev. E 74, 016122 (2006).

[21] S. Pradhan and A. Hansen, Phys. Rev. E 72, 026111 (2005).

[22] S. Pradhan, A. Hansen, and P. C. Hemmer, Phys. Rev. Lett. 95, 125501 (2005).

[23] R. C. Hidalgo, K. Kovacs, I. Pagonabarraga, and F. Kun, Europhys. Lett. 81, 54005 (2008).

[24] T. Baxevanis and T. Katsaounis, Phys. Rev. E 75, 046104 (2007).

[25] F. Raischel, F. Kun, and H. J. Herrmann, Phys. Rev. E 74, 035104(R) (2006).

[26] K. Kóvacs, S. Nagy, R. C. Hidalgo, F. Kun, H. J. Herrmann, and I. Pagonabarraga, Phys. Rev. E 77, 036102 (2008).

[27] F. Raischel, F. Kun, and H. J. Herrmann, Phys. Rev. E 77, 046102 (2008).

[28] U. Divakaran and A. Dutta, Phys. Rev. E 75, 011117 (2007).

[29] U. Divakaran and A. Dutta, Phys. Rev. E 75, 011109 (2007).

[30] R. C. Hidalgo, Y. Moreno, F. Kun, and H. J. Herrmann, Phys. Rev. E 65, 046148 (2002).

[31] R. C. Hidalgo, S. Zapperi, and H. J. Herrmann, J. Stat. Mech. (2008) P01004.

[32] S. Zapperi, A. Vespignani, and H. E. Stanley, Nature (London) 388, 658 (1997).

[33] F. Kun, S. Zapperi, and H. J. Herrmann, Eur. Phys. J. B 17, 269 (2000).

[34] R. C. Hidalgo, F. Kun, and H. J. Herrmann, Phys. Rev. E 64, 066122 (2001).

[35] M. Abramowitz and I. A. Stegun, Handbook of Mathematical Functions (Dover, New York, 1968).

[36] S. Pradhan, P. Bhattacharyya, and B. K. Chakrabarti, Phys. Rev. E 66, 016116 (2002).

[37] P. C. Hemmer and S. Pradhan, Phys. Rev. E 75, 046101 (2007). 